\title{
Dynamic Facilitation Picture of a Higher-Order Glass Singularity
}

\author{
Mauro Sellitto, ${ }^{1}$ Daniele De Martino, ${ }^{2}$ Fabio Caccioli, ${ }^{2}$ and Jeferson J. Arenzon ${ }^{3}$ \\ ${ }^{1}$ United World College in Mostar, Španski trg 1, 88000 Mostar, Bosnia and Herzegovina \\ ${ }^{2}$ International School for Advanced Studies, via Bonomea 265, I-31136 Trieste, Italy \\ ${ }^{3}$ Instituto de Física and INCT-Sistemas Complexos, Universidade Federal do Rio Grande do Sul, \\ CP 15051, 91501-970 Porto Alegre RS, Brazil
}

(Received 1 October 2010; revised manuscript received 25 November 2010; published 29 December 2010)

\begin{abstract}
We show that facilitated spin mixtures with a tunable facilitation reproduce, on a Bethe lattice, the simplest higher-order singularity scenario predicted by the mode-coupling theory (MCT) of liquid-glass transition. Depending on the facilitation strength, they yield either a discontinuous glass transition or a continuous one, with no underlying thermodynamic singularity. Similar results are obtained for facilitated spin models on a diluted Bethe lattice. The mechanism of dynamical arrest in these systems can be interpreted in terms of bootstrap and standard percolation and corresponds to a crossover from a compact to a fractal structure of the incipient spanning cluster of frozen spins. Theoretical and numerical simulation results are fully consistent with MCT predictions.
\end{abstract}

DOI: 10.1103/PhysRevLett.105.265704

PACS numbers: 64.70.qj, 64.70.P-, 68.35.Rh

Although the glassy state of matter has been a long-time fascinating topic for physicists, it still presents several mysterious aspects [1]. Perhaps, the most controversial one is the very nature of the glass transition: that is the question of whether vitrification is a purely dynamical process or rather the (dynamical) manifestation of a genuine thermodynamic amorphous phase. In spite of much progress (for reviews, see [2-6]) the problem remains widely open. On one hand, there are notorious experimental and computational difficulties related to the exceedingly long equilibration times of macroscopic samples, and to the possibility of detecting unambiguously the elusive "amorphous order." On the other hand, theoretical modeling of glassy systems has made clear that slow relaxation processes are ubiquitous and may result from very distinct mechanisms.

In a situation in which it is unknown to what extent thermodynamics and dynamics are intertwined, it would be particularly advantageous from a methodological point of view to identify those peculiar glassy features that can be reproduced with no reference to specific energetic interactions. In this respect, facilitated spin models first introduced by Fredrickson and Andersen are particularly useful [7], as they are constructed in such a way as to have a manifestly uninteresting thermodynamics. Hence they allow to clearly disentangle, albeit in an arguably artificial way, dynamic aspects from static ones.

The first assumption of the dynamic facilitation approach is that, on a suitable coarse-grained length scale, one can model the structure of a liquid by an assembly of high (low) density mesoscopic cells which have no static interaction. Binary spin variables, taking on value \pm 1 , can be simply assigned to these cells. The next crucial step is to postulate that there exists a time scale over which the effective microscopic dynamics takes a deceptively simple form: local changes in cells structure occur if and only if there is a sufficiently large number, say $f$, of nearby lowdensity cells ( $f$ is called facilitation parameter). The latter assumption is actually difficult to derive by analytical means. Nevertheless, it can be justified on physical grounds: it mimics the cage effect and gives arise to a variety of remarkable, and sometimes unexpected, glassy features, even if the thermodynamics is completely trivial [5]. Although this line of research has been pursued very actively in recent years, little attention has been paid to the possibility of reproducing, within this framework, more complex types of glassy behavior (for some exceptions, see $[8,9])$. In fact, even simple schematic mode-coupling theory (MCT) models predict the occurrence of topologically stable singularities of higher complexity [2]. Liquids confined in a disordered porous matrix [10] and attractive colloids $[11,12]$ are some examples in which these scenarios have been recently observed. Solvable microscopic realizations of such systems would be highly valuable both for a deeper understanding of complex glassy features and for clarifying the limits of MCT. This is quite a delicate issue, however, as mean-field disordered systems which are supposed to be exactly described by MCT are spoiled by finite-size effects and preasymptotic corrections so strong as to prevent a direct observation of MCT predictions $[13,14]$. For this reason, we follow here an alternative route.

Motivated by recent findings on the annealed-quenched mixtures [10], we have generalized the dynamic facilitation approach by allowing for an inhomogeneous distribution of facilitation or, equivalently, as we shall see, of lattice connectivity. While the latter may originate from a geometrically disordered environment, e.g., a porous matrix, the former can be thought of as resulting from the coexistence of different length scales in the problem, e.g., 
mixtures of more or less mobile molecules or polymers with small and large size. By doing so, we show that facilitated spin models on Bethe lattice provide a close microscopic realization of the simplest higher-order bifurcation singularity scenario predicted by MCT, in which two liquid-glass transition lines (of types $A$ and $B$ ) join smoothly at a common end point.

The model.-Facilitated spin models consist of $N$ noninteracting spins $\sigma_{i}= \pm 1, i=1, \ldots, N$ with Hamiltonian $\mathcal{H}=-\frac{h}{2} \sum_{i=1}^{N} \sigma_{i}$, evolving with a Metropolis-like dynamics: at each time step a randomly chosen spin is flipped with transition probability: $w\left(\sigma_{i} \rightarrow-\sigma_{i}\right)=$ $\min \left\{1, e^{-h \sigma_{i} / k_{B} T}\right\}$, if and only if at least $f$ of its $z$ neighboring spins are in the state -1 (hereafter $h / k_{B}=1$ ). On a Bethe lattice, the dynamics can be characterized by exploiting the relation with the bootstrap percolation $[15,16]$. Disregarding the less interesting noncooperative case, $f=1$, in which there is no transition, one can distinguish two cases. (i) For $z-1>f>1$, the system undergoes a dynamical arrest: below a certain temperature $T_{c}$, the fraction of frozen spins $\Phi$, which plays the role of the nonergodicity parameter in MCT, jumps from zero to a finite value. This corresponds to the sudden emergence of a giant cluster of frozen spins (i.e., spins that are surrounded by more than $f$ neighboring 1 spins) with compact structure. This dynamic transition has a very peculiar hybrid nature: it is discontinuous and, at the same time, has diverging fluctuations as in continuous phase transitions. The geometric origin of this behavior has been understood quite in detail as being related to the divergence of the size of corona clusters near the transition $[17,18]$. Several results [16], including those related to large scale cooperative rearrangements responsible for slow dynamics [19], have suggested a strong analogy with MCT. (ii) For $f=z-1, z$ the transition is continuous as bootstrap percolation is equivalent to conventional percolation [15], and for this reason it has attracted less interest. The facilitated spin dynamics of these systems has never been explored to our knowledge.

To study the crossover between integer values of $f$ we introduce a new facilitated spin model in which the facilitation strength can be continuously tuned. This is obtained by making the facilitation parameter a lattice site dependent quenched random variable, in close analogy with the bootstrap percolation problem studied by Branco [20]. For concreteness, we shall consider a Bethe lattice with coordination number $z=4$, in which the facilitation $f_{i}$ is chosen from the probability distribution:

$$
P\left(f_{i}\right)=(1-q) \delta_{f_{i}, 2}+(q-r) \delta_{f_{i}, 3}+r \delta_{f_{i}, 4},
$$

with $1 \geq q \geq r \geq 0$. By tuning $q$ and $r$ we can thus explore the discontinuous-continuous glass transition crossover.

Exact results.-The treelike structure of the Bethe lattice allows for an exact calculation of the phase diagram. Following Refs. $[15,16,20]$ we get:

$$
T_{c}(q)= \begin{cases}\frac{1}{\log (8-12 q)}, & \text { if } 0 \leq q \leq 1 / 2 \\ -\frac{1}{\log (3 q-1)}, & \text { if } 1 / 2<q<2 / 3\end{cases}
$$

The phase diagram is depicted in Fig. 1, and comprises two lines that smoothly join at $q=1 / 2$. As expected, the introduction of less facilitated spins increases the glass transition temperature, as compared to the pure case, $q=0$ [16]. The precise nature of the two glass transitions depends on the behavior of $\Phi$ near $T_{c}$. Denoting with $p=1 /\left(1+e^{-1 / T}\right)$ the probability that a spin is 1 in thermal equilibrium, we find

$$
\begin{aligned}
\Phi= & p\left[x^{3}(4-3 x)+6 q x^{2}(1-x)^{2}+4 r x(1-x)^{3}\right] \\
& +(1-p)\left[y^{3}(4-3 y)+6 q y^{2}(1-y)^{2}+4 r y(1-y)^{3}\right],
\end{aligned}
$$

where $y=p\left[x^{3}+3(1-q) x^{2}(1-x)+3 r x(1-x)^{2}\right]$, and $x=\left[3 p(1-2 q)+\sqrt{9 p^{2}-8 p+12 q p(1-p)}\right] / 2 p(2-$ $3 q$ ). The behavior of $\Phi$ as a function of the temperature is shown in the inset of Fig. 1, for several values of $q$ and with $r=10^{-3}$. Notice that $\Phi$ generally depends on $r$ while the phase diagram does not. For $0 \leq q \leq 1 / 2$, $\Phi$ jumps to a finite value $\Phi_{c}=\Phi\left(T_{c}\right)$ on the transition line, meaning that the infinite cluster of frozen spins has a compact structure. This structure turns out to be quite resilient against random inhomogeneities in the facilitation strength, in a rather large range of $q$. The critical exponent $\beta$ associated to the order parameter $\Phi$ is obtained by expanding the above equations in the small parameter $\epsilon=T-T_{c}$. As expected, we find $\Phi-\Phi_{c} \sim \epsilon^{\beta}$, with $\beta=1 / 2$, that is the typical square-root dependence well known in MCT and in other systems with hybrid transition. For $1 / 2<q<2 / 3$ the glass transition changes nature as $\Phi$ departs smoothly from zero with a power-law behavior

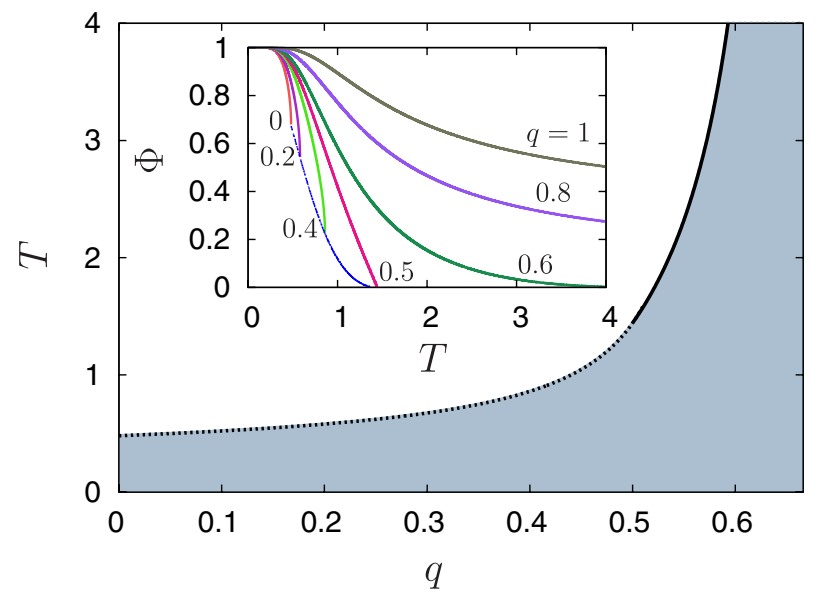

FIG. 1 (color online). Phase diagram for $z=4$ and facilitation as in (1). The dark region is the glassy phase. The dashed (solid) line is the discontinuous (continuous) transition. Inset: Fraction of frozen spins vs temperature for several values of $q$ and $r=$ $10^{-3}$. Below $q=1 / 2, \Phi$ jumps to a finite value at the transition which is represented by the dotted line. 
$\Phi \sim \epsilon^{\beta}$, and the correspondence with standard percolation suggests that the giant cluster of frozen spins has a fractal structure. Interestingly, in this case $\beta$ depends on $r$ : for $r=0$ one has $\beta=2$, while as soon as a negligibly small amount of spins with $f_{i}=4$ is introduced into the system, $r>0$, one has $\beta=1$. The robustness of the latter behavior reflects the fact that the mass of the fractal cluster of frozen spins is essentially dominated by the dangling ends, that is those parts of the cluster which are connected to the backbone by a single site. Only when $r=0$, dangling ends are completely removed from the infinite cluster and the exponent changes to $\beta=2$ [20]. Hence, the general scenario emerging for an arbitrary ternary mixture is that of two distinct glass transitions with $\beta=1 / 2$ (for the discontinuous case), and $\beta=1$ (for the continuous one). These critical exponents reproduce exactly the MCT results for the $F_{12}$ schematic model [2], in which the memory kernel takes the form $m_{12}=v_{1} \phi(t)+v_{2} \phi^{2}(t)$.

Numerical simulations. - We now turn to numerical simulation to explore those features of equilibrium relaxation which are relevant for a comparison with MCT. Testing MCT in systems which are described by a somewhat ad hoc kinetic rule is particularly interesting because it allows us to probe the degree of universality of MCT results beyond the context (the actual Newtonian or Brownian liquid dynamics) and the approximations in which they were originally derived. The dynamics of facilitated spin systems is conveniently characterized by the persistence $\phi(t)$, i.e., the probability that a spin has never flipped between times 0 and $t$. The long-time limit of $\phi(t)$, which plays the role of the Edwards-Anderson parameter in spin glasses, is directly related to the fraction of frozen spins $\Phi=\lim _{t \rightarrow \infty} \phi(t)$, which is known to describe broken ergodicity. Simulation results for the persistence are shown in Fig. 2 for various $q$, above and below the glass transition temperature $T_{c}(q)$. To avoid dynamic reducibility problems the ternary mixture has a negligibly small fraction of spins with $f=4$, typically $r=10^{-3}$. We see that inside the glassy phase, the persistence attains a finite plateau which is in excellent agreement with the value of $\Phi$ analytically computed in the previous section. On approaching $T_{c}(q)$ from above and for $q<1 / 2$ we find typical signatures of MCT: two-step decay of $\phi(t)$, late stage stretched exponential relaxation, and power-law form of equilibration time. When $q \rightarrow 1 / 2$ (and so $\Phi_{c} \rightarrow 0$ ), the range over which the time-temperature superposition principle holds shrinks, and consequently no simple scaling form of relaxation data at various temperature can be found in this limit.

To make a more stringent test of MCT we now examine in detail the critical dynamics on the glass transition lines, for which MCT predicts distinctive patterns of universal critical behavior. When the system is relaxing exactly at the critical temperature $T_{c}(q)$, MCT predicts the powerlaw decay, $\phi(t)-\phi(\infty) \sim t^{-a(q)}$, irrespective of whether the long-time limit of persistence is finite or zero. Figure 3

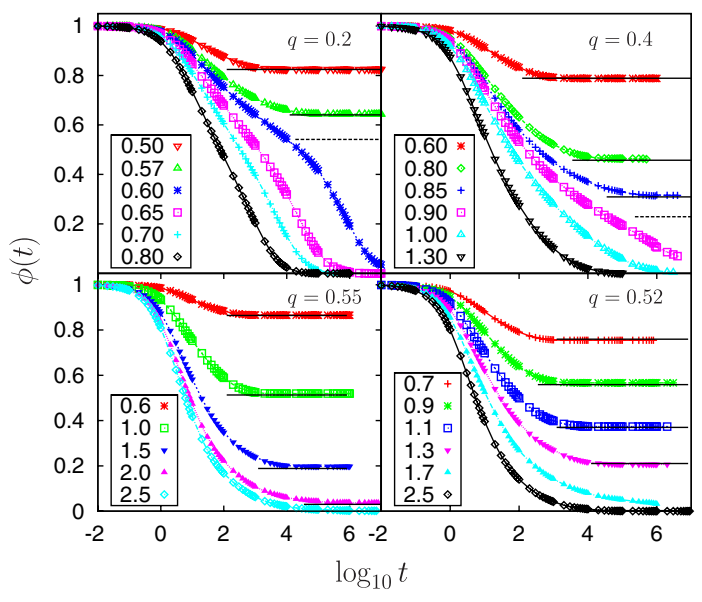

FIG. 2 (color online). Persistence vs time for several temperatures, indicated in the key, and values of $q$. The transition is discontinuous (continuous) for $q$ smaller (larger) than $1 / 2$. We use $r=10^{-3}$, except for $q=0.52$ where $r=0$. The horizontal lines show the theoretical prediction for the plateau heights (the dashed one is the critical plateau at $T=T_{c}$ ). The simulations are performed on lattices with $N=10^{5}$ to $5 \times 10^{5}$ sites and averaged over 2-20 samples.

shows that indeed this power-law behavior is very well obeyed. A further remarkable prediction of MCT is that the exponent $a(q)$ is intimately related, in a way that depends on the nature of the glass transition, to the power-law exponent describing the divergence of the characteristic time $\tau$ on approaching the plateau at $\Phi_{c}$ (the so-called $\beta$-relaxation regime): when temperature gets closer to $T_{c}(q)$ one has $\tau \sim \epsilon^{-1 / 2 a(q)}$ for the discontinuous transition, and $\tau \sim \epsilon^{-1 / a(q)}$ for the continuous one. These two distinct behaviors are tested in the inset of Fig. 3 by assuming as a reasonable definition of the $\beta$-relaxation time $\tau$, the integral of $\phi(t)$ from zero up to the time $t^{*}$

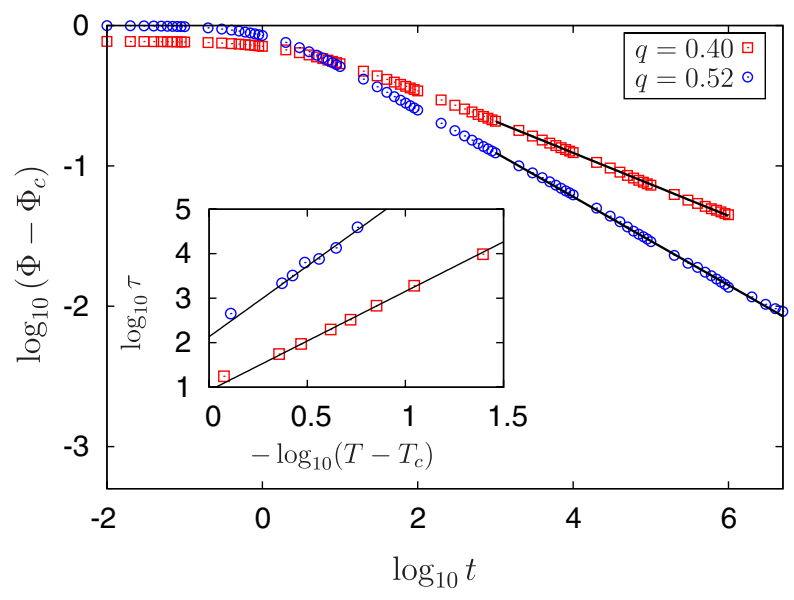

FIG. 3 (color online). Equilibrium relaxation at criticality for discontinuous $(q=0.4)$ and continuous $(q=0.52)$ glass transition. Solid lines are power-law fits with exponent $a=0.224$ and $a=0.315$. Inset: $\beta$-relaxation time $\tau$ vs temperature $T$. Solid lines are power laws with exponent $1 / 2 a$ and $1 / a$, respectively. 
such that $\phi\left(t^{*}\right)=\Phi_{c}$. The estimated $\tau$ behaves as a power law of $\epsilon$ near $T_{c}$ and, more importantly, the exponents perfectly agree with MCT, for both the discontinuous and continuous glass transition. The departure from the plateau, the so-called $\alpha$-relaxation regime, is also consistent with the exponent $b$ obtained from the universal MCT relation $\quad \Gamma(1-a)^{2} / \Gamma(1-2 a)=\Gamma(1+b)^{2} / \Gamma(1+2 b)$, though this regime is much more difficult to analyze due the exceedingly long time scales of numerical simulations.

The excellent agreement we found with MCT could not be obviously anticipated by looking at the "simple" facilitation rule and is all the more remarkable by considering that (i) no exact mode-coupling equation holds for facilitated spin models [21], and (ii) the facilitation rule is admittedly quite remote from the actual microscopic liquid dynamics. Furthermore, our results imply that no thermodynamic transition is actually needed to observe a complex glassy scenario, and suggest that the difficulty to observe MCT predictions in mean-field spin glasses, is generally related to the fact that the dynamic and static transition properties in these systems are tightly twisted.

Conclusions.-To summarize, we have extended the dynamic facilitation approach to glassy systems by introducing facilitated spin mixtures which exhibit a crossover from a discontinuous to a continuous glass transition, and shown that they provide a close microscopic realization of the simplest higher-order glass singularity predicted by MCT. Although we focused on Bethe lattice with fixed connectivity and random distribution of facilitation, it is worth remarking that one can consider an equivalent variant, in which the Bethe lattice is diluted and the facilitation is uniform. For example, results qualitatively similar to those reported above can be obtained when the local lattice connectivity $z_{i}$ is distributed according to $P\left(z_{i}\right)=$ $(1-q) \delta_{z_{i}, 4}+(q-r) \delta_{z_{i}, 3}+r \delta_{z_{i}, 2}$, and $f_{i}=2$ on every site. In this case, less connected sites turn out to have a smaller probability to flip, just as if they were less facilitated. We thus expect, and indeed find, that both variants give essentially the same results. Thus, the crossover between the two glass transitions is obtained by varying either the local connectivity or the facilitation strength, and corresponds to a passage from bootstrap to standard percolation transition. Consistently with MCT predictions, the critical dynamics on both glass transition lines is characterized by power-law decays with exponents closely related to those describing the divergence of the $\beta$-relaxation time. We also mention that when a fraction of spins is overfacilitated as compared to the case $f_{i}=2$, i.e., when $P\left(f_{i}\right)=(1-q) \delta_{f_{i}, 2}+(q-r) \delta_{f_{i}, 1}+r \delta_{f_{i}, 0}$, there is only a discontinuous glass transition line.

Our work can be naturally developed in several directions. The most obvious one consists in considering kinetically constrained particle mixtures, what would be a much closer microscopic realization of a fluid confined in a disordered porous matrix. The comparison with geometrically constrained lattice glass mixtures [22] would then allow for a better understanding of the distinguishing features of glassy systems with and without an ideal Kauzmann transition. We then expect that introducing a suitable static attraction [8] can account for nonmonotonic dependence of relaxation time on attraction strength and, possibly, for phase reentrant behavior. Finally, the existence of kinetic models with a glass transition in $d=2$ [23], suggests that there is no a priori limitation to extending our approach to finite dimensions.

M. S. warmly thanks Professor Götze for kindly clarifying some issues related to MCT, and the Brazilian agency CAPES for a PVE grant. D. D. M. and F. C. acknowledge MIUR for Grant No. 2007JHLPEZ. J. J. A. is partially supported by the Brazilian agencies CNPq (Prosul490440/2007) and FAPERGS.

[1] J. Langer, Phys. Today 60, No. 2, 8 (2007).

[2] W. Götze, Complex Dynamics of Glass-Forming Liquids (Oxford University Press, Oxford, 2009).

[3] K. Binder and W. Kob, Glassy Materials and Disordered Solids (World Scientific, Singapore, 2005).

[4] G. Biroli and J.P. Bouchaud, arXiv:0912.2542.

[5] F. Ritort and P. Sollich, Adv. Phys. 52, 219 (2003).

[6] J.P. Garrahan and D. Chandler, Annu. Rev. Phys. Chem. 61, 191 (2010).

[7] G. H. Fredrickson and H. C. Andersen, Phys. Rev. Lett. 53, 1244 (1984).

[8] P. L. Geissler and D. R. Reichman, Phys. Rev. E 71, 031206 (2005).

[9] A. J. Moreno and J. Colmenero, J. Chem. Phys. 125, 016101 (2006).

[10] V. Krakoviack, Phys. Rev. Lett. 94, 065703 (2005); Phys. Rev. E 75, 031503 (2007).

[11] K. Dawson, G. Foffi, M. Fuchs, W. Götze, F. Sciortino, M. Sperl, P. Tartaglia, Th. Voigtmann, and E. Zaccarelli, Phys. Rev. E 63, 011401 (2000).

[12] K. N. Pham et al., Science 296, 104 (2002).

[13] C. Brangian, W. Kob, and K. Binder, J. Phys. A35, 191 (2002).

[14] T. Sarlat, A. Billoire, G. Biroli, and J.-P. Bouchaud, J. Stat. Mech. (2009) P08014.

[15] J. Chalupa, P. L. Leath, and G. R. Reich, J. Phys. C 12, L31 (1979).

[16] M. Sellitto, G. Biroli, and C. Toninelli, Europhys. Lett. 69, 496 (2005).

[17] J. M. Schwarz, A. J. Liu, and L. Q. Chayes, Europhys. Lett. 73, 560 (2006).

[18] S. N. Dorogovtsev, A. V. Goltsev, and J.F.F. Mendes, Phys. Rev. Lett. 96, 040601 (2006).

[19] A. Montanari and G. Semerjian, Phys. Rev. Lett. 94, 247201 (2005); (unpublished).

[20] N. S. Branco, J. Stat. Phys. 70, 1035 (1993).

[21] S. J. Pitts, T. Young, and H. C. Andersen, J. Chem. Phys. 113, 8671 (2000).

[22] R. K. Darst, D. R. Reichman, and G. Biroli, J. Chem. Phys. 132, 044510 (2010).

[23] C. Toninelli, G. Biroli, and D. S. Fisher, Phys. Rev. Lett. 96, 035702 (2006). 